\title{
FGF-21 as a novel metabolic regulator
}

\author{
Alexei Kharitonenkov, ${ }^{1}$ Tatiyana L. Shiyanova, ${ }^{1}$ Anja Koester, ${ }^{1}$ \\ Amy M. Ford, ${ }^{1}$ Radmila Micanovic, ${ }^{1}$ Elizabeth J. Galbreath, ${ }^{1}$ George E. Sandusky, ${ }^{1}$ \\ Lisa J. Hammond, ${ }^{1}$ Julie S. Moyers, ${ }^{1}$ Rebecca A. Owens, ${ }^{1}$ Jesper Gromada, ${ }^{2}$ \\ Joseph T. Brozinick, ${ }^{1}$ Eric D. Hawkins, ${ }^{1}$ Victor J. Wroblewski, ${ }^{1}$ De-Shan Li, ${ }^{1}$ \\ Farrokh Mehrbod, ${ }^{1}$ S. Richard Jaskunas, ${ }^{1}$ and Armen B. Shanafelt ${ }^{1}$
}

${ }^{1}$ Lilly Research Laboratories, Division of Eli Lilly and Co., Indianapolis, Indiana, USA. ${ }^{2}$ Lilly Research Laboratories, Hamburg, Germany.

\begin{abstract}
Diabetes mellitus is a major health concern, affecting more than $5 \%$ of the population. Here we describe a potential novel therapeutic agent for this disease, FGF-21, which was discovered to be a potent regulator of glucose uptake in mouse 3T3-L1 and primary human adipocytes. FGF-21-transgenic mice were viable and resistant to diet-induced obesity. Therapeutic administration of FGF-21 reduced plasma glucose and triglycerides to near normal levels in both $o b / o b$ and $d b / d b$ mice. These effects persisted for at least 24 hours following the cessation of FGF-21 administration. Importantly, FGF-21 did not induce mitogenicity, hypoglycemia, or weight gain at any dose tested in diabetic or healthy animals or when overexpressed in transgenic mice. Thus, we conclude that FGF-21, which we have identified as a novel metabolic factor, exhibits the therapeutic characteristics necessary for an effective treatment of diabetes.
\end{abstract}

\section{Introduction}

While the majority of the 22 known members of FGF family have been primarily associated with mitosis, development, transformation, angiogenesis, and survival (1-5), recent data shows that they may play important roles in defining and regulating functions of some endocrine-relevant tissues and organs, as well as modulating various metabolic processes. For example, FGF-10 is implicated in the differentiation processes in white adipose tissue (6) and pancreas $(7,8)$, while FGF-16 (9) is considered to be a specific factor for brown adipocytes. Another recently characterized molecule, FGF-19 $(10,11)$, has been shown to cause resistance to diet-induced obesity and insulin desensitization and to improve insulin, glucose, and lipid profiles in diabetic rodents. Since these effects, at least in part, are mediated through the observed changes in metabolic rates, FGF-19 can be considered as a regulator of energy expenditure $(12,13)$.

FGFs modulate cellular activity via at least 5 distinct subfamilies of high-affinity FGF receptors (FGFRs): FGFR-1, -2, -3, and -4, all with intrinsic tyrosine kinase activity and, except for FGFR-4, multiple splice isoforms (1-3); and FGFR-5 $(14,15)$, which lacks an intracellular kinase domain. There is growing evidence that FGFRs can be important for regulation of glucose and lipid homeostasis. The overexpression of a dominant negative form of FGFR-1 in $\beta$ cells leads to diabetes in mice, which thus implies that proper FGF signaling is required for normal $\beta$ cell function and glycemia maintenance (16). FGFR-2 appears to be a key molecule during pancreatic development (17-19). Moreover, FGFR-4 has been implicated in cholesterol metabolism and bile acid synthesis (20).

FGF-21 (21) is a novel member of FGF family. It is preferentially expressed in liver, but an exact knowledge of FGF-21 bioactivity and its mode of action have been lacking to date. Here we show

Nonstandard abbreviations used: BAT, brown adipose tissue; BMP-9, bone morphogenic protein-9; $\mathrm{EC}_{50}, 50 \%$ effective concentration; FGFR, FGF receptor; FRS-2, FGFR substrate-2; GLP-1, glucagon-like peptide-1; GLUT, glucose transporter; HFHC, high-fat/high-carbohydrate diet; HMEC, primary human mammary epithelial cell; HUVEC, human umbilical vein endothelial cell; OGTT, oral glucose tolerance test; PCNA, proliferative cell nuclear antigen; ZDF, Zucker diabetic fatty.

Conflict of interest: The authors have declared that no conflict of interest exists.

Citation for this article: J. Clin. Invest. 115:1627-1635 (2005).

doi:10.1172/JCI23606. that FGF-21 is a potent activator of glucose uptake on adipocytes, protects animals from diet-induced obesity when overexpressed in transgenic mice, and lowers blood glucose and triglyceride levels when therapeutically administered to diabetic rodents. Thus, we believe our report to be the first to document a clear biological function of this protein and its potential therapeutic application.

\section{Results}

Identification of FGF-21 in vitro bioactivity. Using a glucose uptake assay to search for novel proteins with therapeutic potential to treat diabetes mellitus, we found that human recombinant FGF-21 stimulated glucose incorporation in differentiated mouse 3T3-L1 adipocytes, as well as in human primary adipocytes after 24-hour treatment of the cells with the protein (Figure 1, A and B). Since FGF-21 did not induce glucose uptake in undifferentiated 3T3-L1 fibroblasts, human primary preadipocytes, muscle L6-glucose transporter4 myc (L6-GLUT-4myc) myoblasts and myotubes (22), or liver clone 9 cells, the FGF-21 effect appeared to be adipocyte specific.

The effects of FGF-21 on glucose uptake in adipocytes were insulin independent, additive to the activity of insulin upon cotreatment (Figure 1C), and not modulated by addition of exogenous heparin. In contrast to the rapid response elicited by insulin, the predominant effect of FGF-21 on glucose uptake required at least 4 hours of cell treatment, and it was substantially diminished in the presence of cycloheximide $(1 \mu \mathrm{g} / \mathrm{ml})$, a protein synthesis inhibitor (23) (Figure 1D). These observations led us to hypothesize that the mode of action for FGF-21 requires transcriptional activation.

To identify a potential mechanism at the molecular level by which FGF-21 increases glucose uptake, we examined whether it modulates the expression levels of the glucose transporters GLUT1 and GLUT4 in 3T3-L1 adipocytes. FGF-21 treatment $(1 \mu \mathrm{g} / \mathrm{ml})$ led to a significant increase in GLUT1 mRNA and protein but not those of GLUT4 (Figure 1, E and F). FGF-21-dependent GLUT1 upregulation was further demonstrated in vivo following a bolus injection in $o b / o b$ mice. Four hours after s.c. administration of FGF-21 (500 $\mu \mathrm{g} / \mathrm{animal})$, an increase in GLUT1 mRNA was specifically detected in white adipose tissue but not in muscle, liver, kidney, and brain (Figure 1G).

Additional studies revealed that early FGF-21-induced signaling in 3T3-L1 adipocytes included heparin-independent tyrosine phos- 


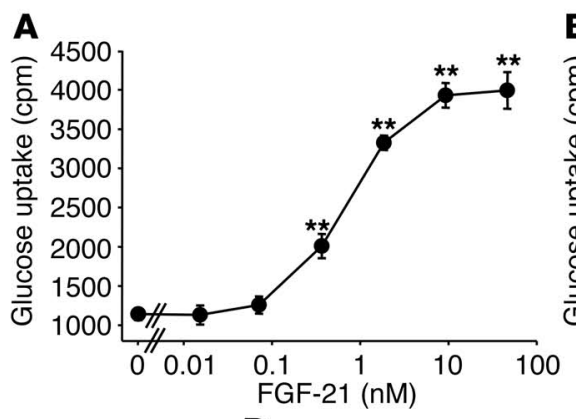

D

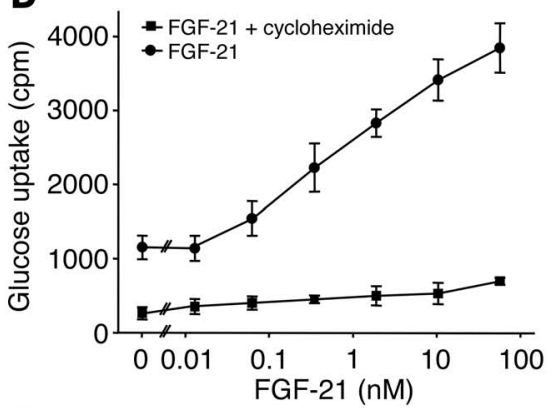

$\mathbf{F}$
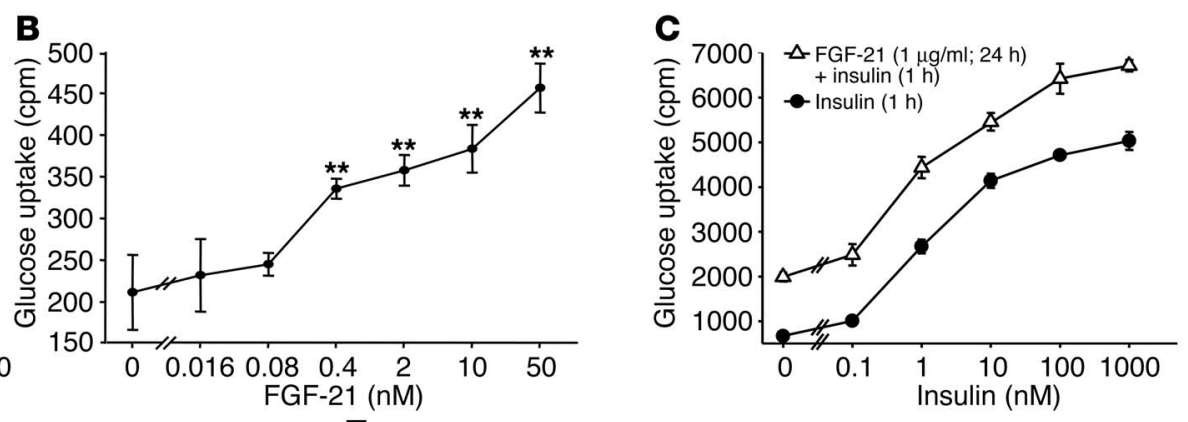

$\mathbf{E}$
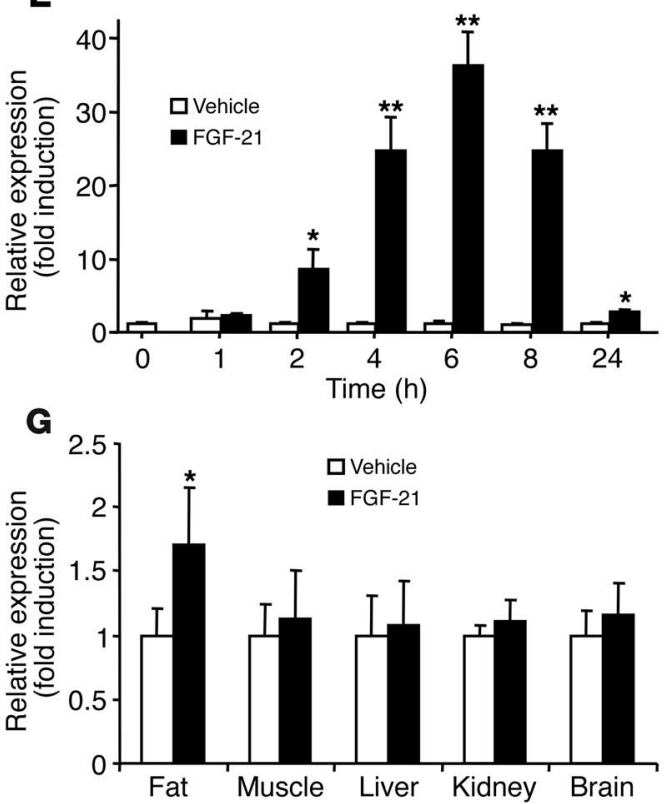

Figure 1

FGF-21 stimulates glucose uptake and modulates GLUT1 expression. The values ( \pm SE) shown are the average of at least 3 independent measurements. ${ }^{*} P<0.02,{ }^{* *} P<0.001$ compared with no stimulation or vehicle control. FGF-21 dose response on 3T3-L1 (A) and human primary adipocytes (B) in glucose uptake assay. (C) FGF-21 augments insulin activity. Cells were pretreated with or without FGF-21 and then stimulated with insulin as indicated. (D) Cycloheximide diminishes FGF-21 bioactivity in glucose uptake assay. 3T3-L1 adipocytes were stimulated with FGF-21 for 24 hours in the presence or absence of cycloheximide. $P<0.001$ at all doses for FGF-21 versus FGF-21 plus cycloheximide stimulations. FGF-21 affects GLUT1 mRNA (E) and protein (F) levels and does not upregulate GLUT4 protein (F) in 3T3-L1 adipocytes (immunoblot). Cells were starved and then stimulated with FGF-21 or vehicle as indicated. Quantitative PCR and immunoblotting analyses were used to measure mRNA and protein levels, respectively. (G) GLUT1 mRNA is upregulated in adipose tissue of FGF-21-injected ob/ob mice. Two groups (5 animals each) of 8-week-old mice were injected s.c. with FGF-21 or vehicle. Quantitative PCR analysis was used to measure mRNA.

phorylation of FGFR substrate-2 (FRS-2), a docking protein linking FGFRs to the Ras/MAPK pathway (24), and transient activation of MAPK (Figure 2A). All these in vitro activities are typically initiated upon activation of FGFR-mediated pathways (25).

FGFR-1 and FGFR-2 may represent FGF-21-corresponding receptors. The sequence characteristics and signaling profile of FGF-21 indicate that its receptor may belong to the FGFR superfamily. The expression of FGFR-1 and FGFR-2, including several splice variants of these receptors, was readily detected in 3T3-L1 cells by RT-PCR and immunoblot analysis. While FGFR-5 was also detected by RT-PCR, significantly lower levels of FGFR-3 and no expression of FGFR-4 were observed. We immunoprecipitated FGFR-1 and FGFR-2 from 3T3-L1 adipocytes with specific antibodies and detected in both cases 2 tyrosine-phosphorylated proteins of approximately $120-150 \mathrm{kDa}$ in FGF-21-stimulated cells (Figure 2B), while no phosphorylation was observed in FGFR-3 immunoprecipitates under similar conditions (data not shown). These tyrosine-phosphorylated bands were later reprobed with anti-FGFR-1 and anti-FGFR-2 antibodies, respectively (Figure 2B), which indicates that they may represent activated forms of FGFR-1 and FGFR-2. Importantly, the observed FGF-21-dependent phosphorylation was adipocyte specific, since it was not detected in 3T3-L1 preadipocytes.

FGF-21 does not induce in vitro mitogenicity. As a class, FGFs are generally known to induce cell proliferation. Therefore, we examined the mitogenic potential of FGF-21 in cells typically sensitive to FGFs. FGF-21 did not induce proliferation of 3T3-L1, NIH 3T3, or BALB/c 3T3 fibroblasts, monkey epithelial 4MBr5 cells, primary human mammary epithelial cells (HMECs), or human umbilical vein endothelial cells (HUVECs), either in the absence or presence of exogenous heparin. In contrast, FGF-7, FGF-1, and FGF-2 stimulated the growth of these cells. Moreover, in costim- 
A

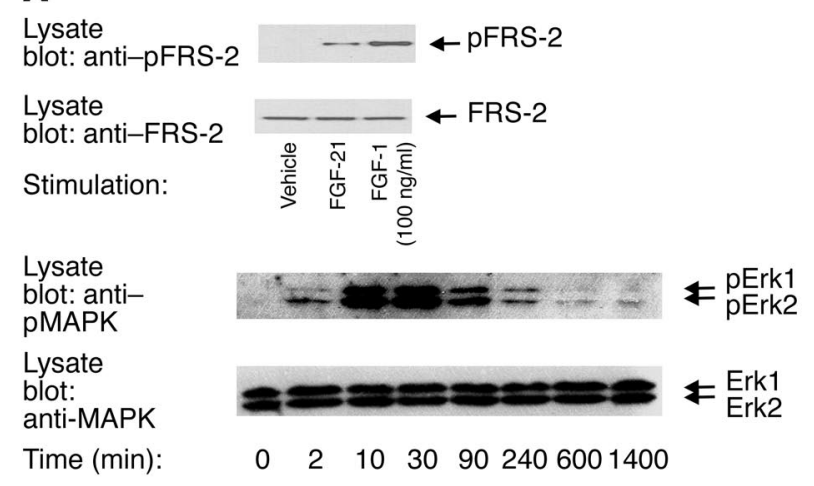

B

IP: anti-FGFR-1

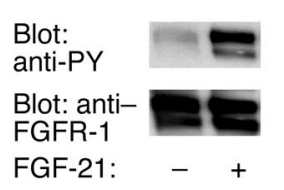

\section{Figure 2}

FGF-21 stimulates phosphorylation in 3T3-L1 adipocytes. (A) FGF-21 induces phosphorylation of MAPK and FRS-2 in 3T3-L1 adipocytes. Upon stimulation, cells were lysed, and phospho-specific antibodies were used to determine phosphorylation of MAPK and FRS-2 in immunoblots. After immunoblots were stripped, anti-MAPK and anti-FRS-2 antibodies were used to confirm that protein loads were equal. For MAPK experiment, cells were stimulated with FGF-21 for the indicated times. For FRS-2 experiment, cells were stimulated with FGF-21 or FGF-1 (positive control). (B) FGF-21 stimulates tyrosine phosphorylation of FGFR-1 and FGFR-2 in 3T3-L1 adipocytes. Cells were stimulated with FGF-21 and lysed. FGFR-1 and FGFR-2 immunoprecipitates were analyzed in immunoblots with anti-phosphotyrosine antibodies. After stripping, anti-FGFR-1 and anti-FGFR-2 antibodies were used to confirm that protein loads were equal. pErk, phospho-Erk; PY, phosphotyrosine.

diabetic or normal animals displayed no evidence of hypoglycemic effects in fed or even in the fasted state (Figure 4, A, C, F, and H).

FGF-21-transgenic mice. In order to evaluate the effect of enforced expression of FGF-21 in vivo, we generated FGF-21-transgenic mice that overexpressed the human protein from the liver using the apoE promoter. As measured by an FGF-21-specific ELISA, the plasma concentrations of FGF-21 in the transgenic animals ranged between 70 and $150 \mathrm{ng} / \mathrm{ml}$.

These FGF-21-transgenic mice were viable and at 2 months of age had glucose levels similar to those of their wild-type littermates. However, at 9 months, differences between transgenic and wildtype mice became apparent. FGF-21-transgenic animals weighed significantly less, had lower fasted glucose levels (Table 1) and less fat in liver, retained more brown adipose tissue, had subcutaneous adipocytes of smaller size (Figure 5, A and B), and exhibited improved glucose clearance and insulin sensitivity relative to control littermates as measured during OGTT (data not shown).

Several members of FGF family have been shown to induce therapeutically undesirable in vivo proliferation of various cell types $(1-5,26)$. Therefore, we examined FGF-21-transgenic mice for their potential to develop tumors throughout their lifespan. As evidenced by histological analysis, transgenic mice overexpressing FGF-21 did not develop liver tumors or show evidence of any other tissue hyperplasia up to 10 months of age (Figure 5C).

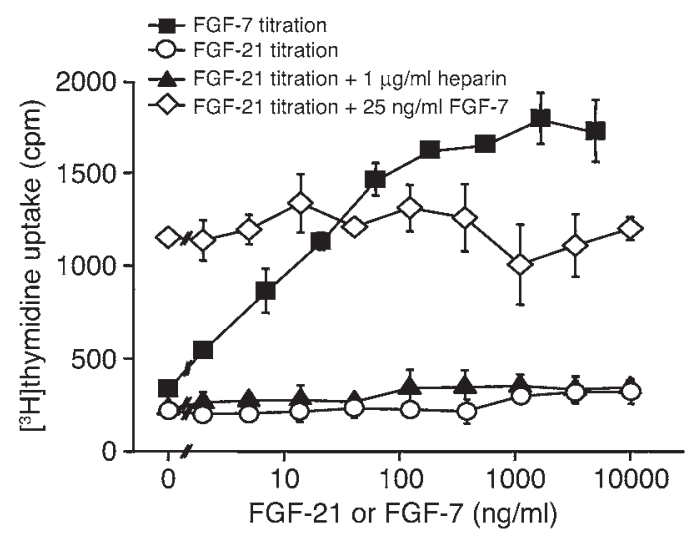

Figure 3

FGF-21 does not induce proliferation and does not block FGF-7dependent mitogenicity on $4 \mathrm{MBr} 5$ cells. Cells were stimulated as indicated with different concentrations of FGF-7, FGF-21, and FGF-21 in the presence of a constant concentration of heparin and FGF-21 in the presence of a constant concentration of FGF-7. 
A

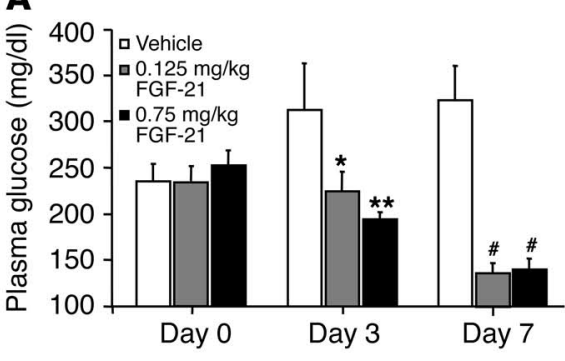

B

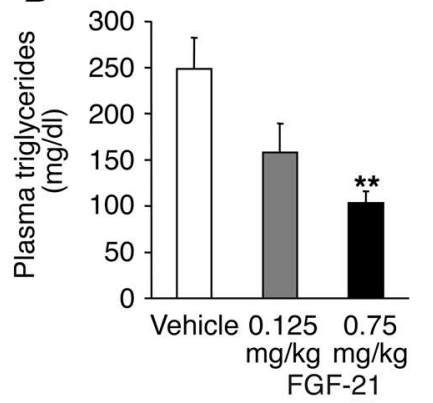

c

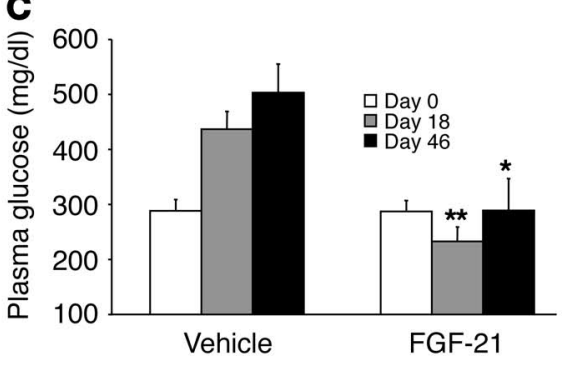

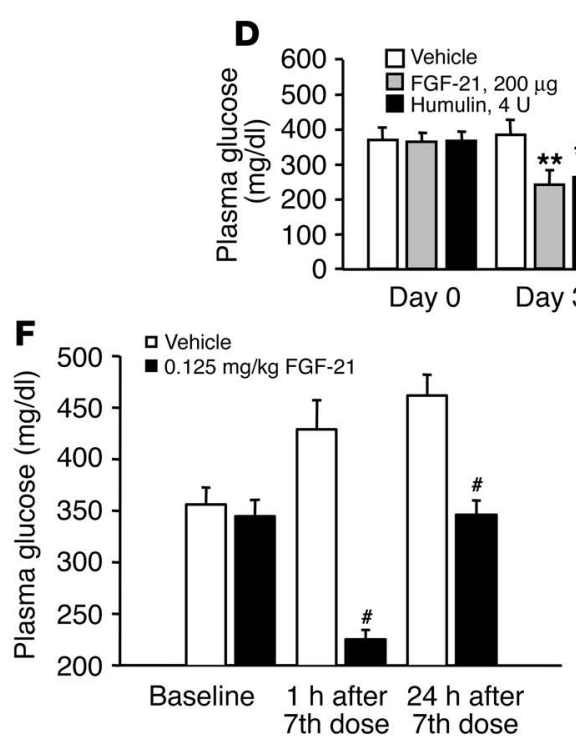

E

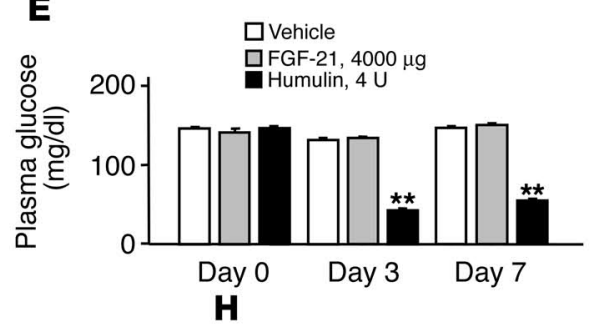

G
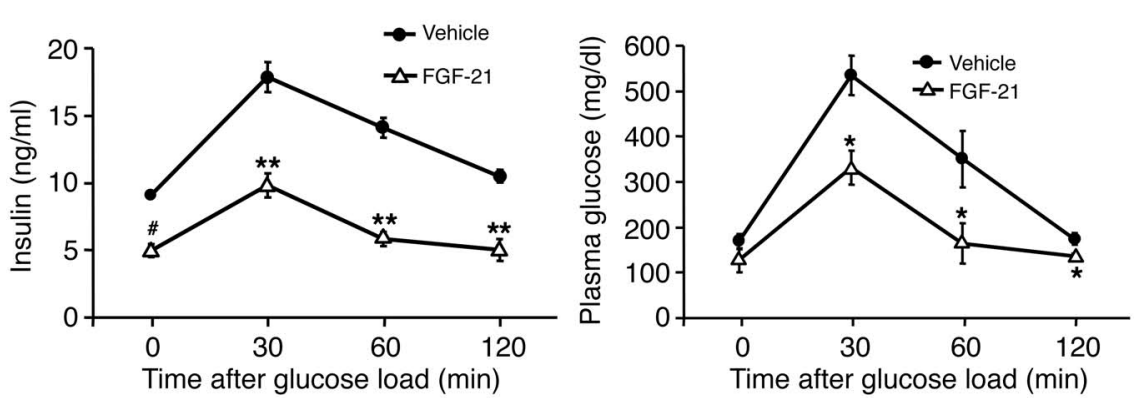

\section{Figure 4}

FGF-21 injection studies in rodents. The values $\left( \pm\right.$ SE) shown are the average of the measurements of at least 5 animals in a group. ${ }^{*} P<0.05$, ${ }^{\star \star} P<0.02$, and ${ }^{\#} P<0.001$ compared with vehicle control. Fed blood glucose (A) and triglyceride levels (B) in ob/ob mice treated with FGF-21. FGF-21 was administered once daily, and blood glucose and triglyceride levels were measured 1 hour after the last injection. (C) Fed blood glucose levels in $d b / d b$ mice at days 18 and 46 during 8-week constant-infusion study. Mice were infused s.c. with $11 \mu \mathrm{g} / \mathrm{kg} / \mathrm{h} \mathrm{FGF-21} \mathrm{using} \mathrm{ALZET}$ minipumps. (D and E) FGF-21 lowers glucose in obese ZDF rats and does not induce hypoglycemia in lean ZDF rats. Fed blood glucose levels were measured in obese (D) and lean (E) ZDF rats that were administered s.c. twice daily with FGF-21, Humulin, or vehicle at indicated total daily doses for 1 week. (F) FGF-21 induces extended lowering of fed blood glucose in ob/ob mice. FGF-21 was administered once daily for 7 days, and blood glucose levels were measured after the last injection at indicated time points. (G and $\mathbf{H})$ FGF-21 affects insulin levels (G) and glucose disposal $(\mathbf{H})$ during OGTT in ob/ob mice. At indicated time points, blood samples were obtained for glucose and insulin measurements.

We challenged FGF-21 transgenic animals by feeding them a high-fat/high-carbohydrate (HFHC) diet for 15 weeks. Intriguingly, FGF-21-transgenic mice consumed almost twice as much food as wild-type littermates when the amounts were normalized to body weights and calculated as the actual amount of food eaten per animal per day (Table 1). Despite the significant increase in caloric intake, they did not gain as much weight as wild-type controls and were also resistant to diet-induced obesity (Figure 6, A and B).

\section{Discussion}

Several secreted polypeptides, including insulin, glucagon-like peptide-1 (GLP-1), adiponectin, and others are ultimately involved in the regulation of glucose homeostasis, which thus makes them clinically relevant pharmacological agents or attractive candidates for novel medicines for the treatment of diabetes mellitus (27). Recent reports on bone morphogenic protein-9 (BMP-9) (28) and FGF-19 (12,13), and our findings with FGF-21, have shown that the other proteins also constitute this list of promising biomolecules.
FGF-21 bioactivity was discovered through a cell-based functional screen aimed at identifying novel secreted molecules that affect glucose uptake on mouse 3T3-L1 adipocytes and was found to be very potent in this assay $\left(\mathrm{EC}_{50}, \sim 0.5 \mathrm{nM}\right.$ ) (Figure $\left.1 \mathrm{~A}\right)$. With comparable potency, FGF-21 was active on differentiated human primary adipocytes (Figure 1B), which indicates that FGF-21 bioactivity is not limited to murine adipocytes.

The follow-up analysis on the initial observation in the glucose uptake assay revealed what we believe to be a novel and unique mechanism of the FGF-21 mode of action. FGF-21 effects appeared to be insulin independent and additive to insulin activity upon coadministration (Figure 1C). FGF-21 needed to be present on cells for several hours to produce a robust response in glucose uptake, and the effect was significantly diminished by the protein synthesis inhibitor cycloheximide (Figure 1D). While insulin is known to work in a rapid, hormone-like manner, we hypothesized that FGF-21 activity is likely to be mediated through changes in gene expression. Indeed, we showed that FGF-21 induced a signifi- 
Table 1

Metabolic parameters in FGF-21-transgenic and control mice

\begin{tabular}{|c|c|c|c|c|c|c|}
\hline & Control (M) & $\operatorname{Tg}(\mathrm{M})$ & $P$ value & Control (F) & $\operatorname{Tg}(F)$ & $P$ value \\
\hline Body weight $(g)^{A}$ & $50.4 \pm 0.7$ & $29.1 \pm 3.37$ & $<0.001$ & ND & ND & ND \\
\hline Fasted glucose $(\mathrm{mg} / \mathrm{dl})^{\mathrm{A}}$ & $102 \pm 5.5$ & $67 \pm 8.1$ & $<0.003$ & ND & ND & ND \\
\hline Food intake (g/g body wt/wk) ${ }^{B}$ & $0.42 \pm 0.02$ & $0.76 \pm 0.03$ & $<0.001$ & $0.55 \pm 0.01$ & $0.88 \pm 0.04$ & $<0.001$ \\
\hline Leptin (ng/ml) ${ }^{\mathrm{B}}$ & $63.0 \pm 1.97$ & $15.6 \pm 4.12$ & $<0.001$ & $61.4 \pm 3.64$ & $18.26 \pm 6.97$ & $<0.001$ \\
\hline Glucagon $(\mathrm{pg} / \mathrm{ml})^{\mathrm{B}}$ & $116 \pm 7.12$ & $93.8 \pm 8.54$ & 0.08 & $141 \pm 9.5$ & $93 \pm 6.55$ & $<0.01$ \\
\hline Body temperature $\left({ }^{\circ} \mathrm{C}\right)^{\mathrm{B}}$ & $35.58 \pm 0.09$ & $35.4 \pm 0.27$ & NS & $36.2 \pm 0.23$ & $36.5 \pm 0.17$ & NS \\
\hline
\end{tabular}

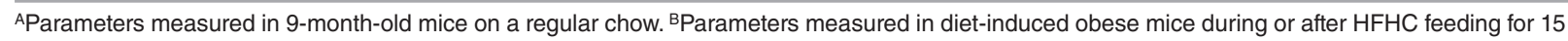
weeks. The values ( \pm SE) shown are the average of the measurements of at least 5 animals in a group. M, males; F, females; Tg, FGF-21-transgenic mice; ND, not determined.

cant upregulation of the insulin-independent glucose transporter GLUT1 in 3T3-L1 adipocytes (Figure 1, E and F) and further confirmed this finding in vivo when we administered FGF-21 to $o b / o b$ mice (Figure 1G). Thus, in contrast to insulin, which is known to function via GLUT4 translocation (29), FGF-21 may primarily act through upregulation of cellular GLUT1 in these cells.

In addition to the changes in GLUT1, we observed a modest reduction in GLUT4 levels after 48 and 72 hours of cell stimulation with FGF-21 (Figure 1F). While FGF-21 stimulation caused a decrease in GLUT4 levels in vitro, it did not appear to negatively impact glucose uptake (Figure 1C), which suggests either that the decrease is not sufficient to affect glucose transport or that other compensatory mechanisms are at work in 3T3-L1 adipocytes. Moreover, we observed improved insulin sensitivity in FGF-21administered diabetic rodents even under enforced overexpression conditions in FGF-21-transgenic mice or after 8 weeks of chronic constant infusion of FGF-21 in $d b / d b$ mice (Figure $4 \mathrm{G}$ and data not shown). Thus, the reduction observed in GLUT4 in 3T3-L1 adipocytes does not appear to be functionally relevant.

FGF-21 is a typical FGF molecule with respect to its ability to stimulate MAPK activation and FRS-2 phosphorylation (25) (Figure 2A). In contrast, while heparin or heparin-like molecules are considered to be essential for the biological activity of proteins of the FGF family (30), none of the FGF-21-induced in vitro responses observed in 3T3-L1 or human adipocytes were heparin regulated. Importantly, FGF-21 appears to be mitogenically inactive in vitro when tested on several otherwise FGF-sensitive cell lines and primary cells (Figure 3). This further establishes FGF-21 as a unique protein within the FGF family, as FGFs are well known to induce proliferation (1-5). Whereas the exact cause of the nonmitogenic character of the in vitro action of FGF-21 is currently unknown, these data help significantly in validating FGF-21 as a potential protein therapeutic.

\section{Figure 5}

Histological analysis of FGF-21-transgenic and FGF-21-infused animals. (A) H\&E staining of brown fat. Notice an increase in intensity of brown fat in the FGF-21-transgenic mouse compared with the wildtype mouse. (B) H\&E staining of subcutaneous white fat. Notice the smaller adipocytes in the FGF-21 mouse compared with the wild type. (C) H\&E staining of livers from FGF-21-transgenic and wild-type mice. There are no differences between the 2. Magnification, $\times 200$ (A-C). (D) PCNA staining of the livers from FGF-21-infused and saline-treated $d b / d b$ mice. PNCA immunostaining shows very low proliferation (less than $5 \%$ ) of hepatocytes (brown staining, arrows) in both the control and treated groups. Magnification, $\times 400$.
The fact that FGF-21 induced tyrosine phosphorylation of FGFR-1 and FGFR-2 (Figure 2B) suggests that these molecules may function as FGF-21 receptors. Although they carry readily detectable and functional FGFR-1 and/or FGFR-2 molecules, it is, however, currently unclear why several FGF-sensitive cells that were tested for FGF-21 bioactivity do not respond to FGF-21 stimulation. Moreover, in preliminary experiments with all commercially available FGFR extracellular domain-Fc fusion proteins (R\&D Systems), we were unable to demonstrate direct interaction between any of these FGFR variants and FGF-21, despite the fact that we clearly observed binding for both FGF-1 and FGF-2 (data not shown). Thus, FGF-21 may be physically interacting with different splice variants of FGFR-1 and FGFR-2 that are induced upon adipocyte differentiation. Alternatively, FGF-21-dependent activation of these receptors may require a fat cell-specific modification or additional cofactor.

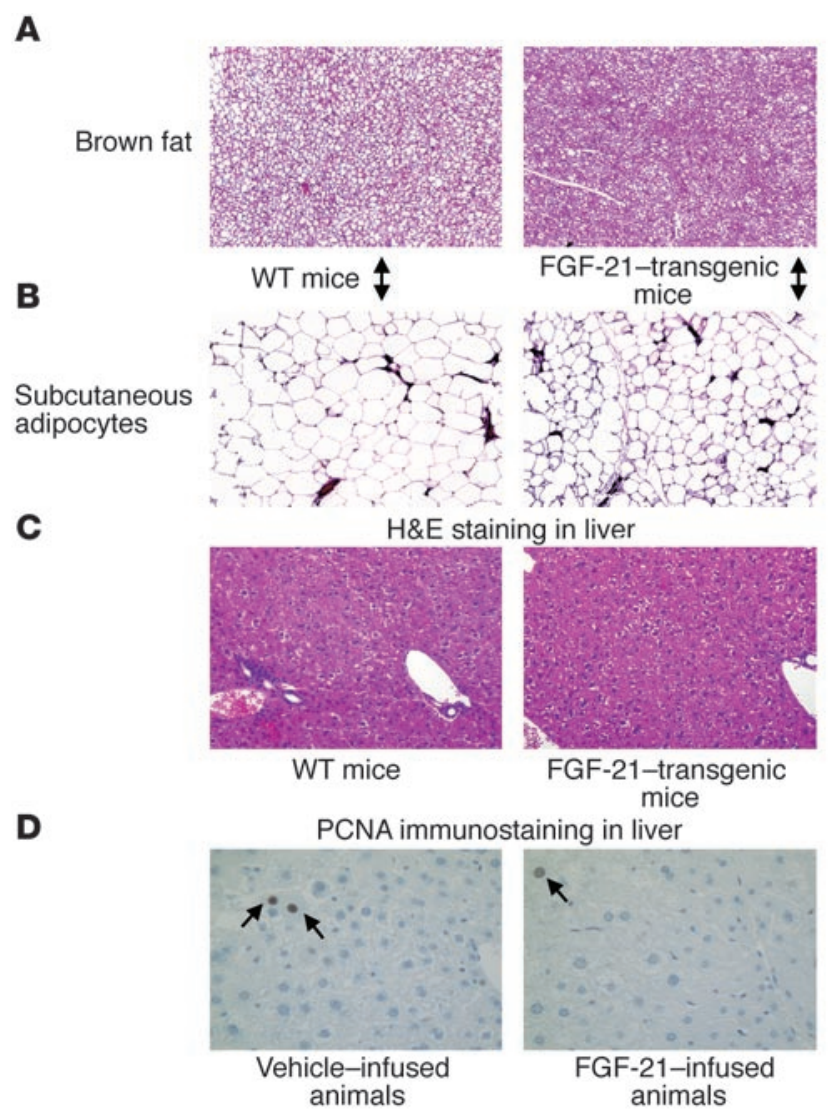



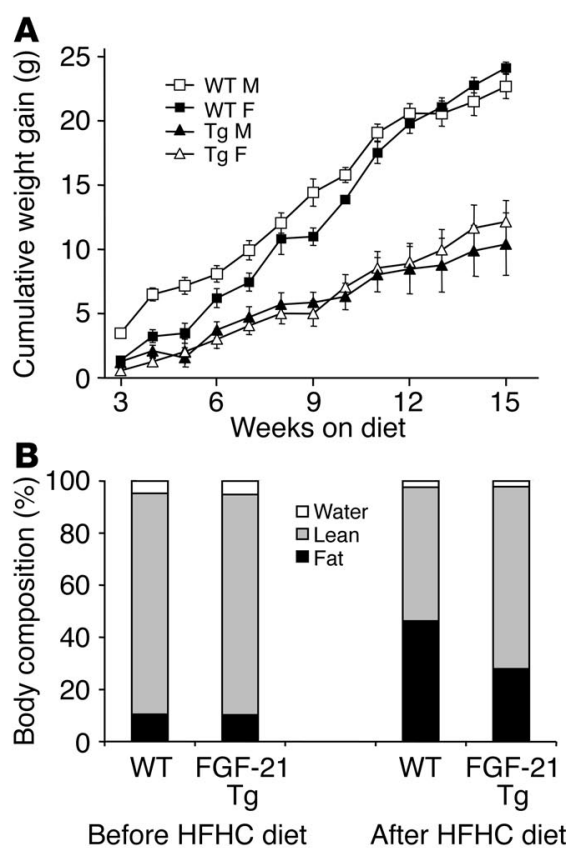

FGF-21 did not stimulate glucose uptake on insulin-sensitive liver clone 9 and muscle L6-GLUT-4myc cells and on 3T3-L1 fibroblasts. Also, we were unable to detect FGF-21 activity in proliferation assays on several cell lines and primary cells of a different nature, which further indicates that FGF-21 effects might be adipocyte specific. Nevertheless, we recently observed a clear FGF-21 response on cells of nonfat origin. Unexpectedly, FGF-21 (1 $\mu \mathrm{g} / \mathrm{ml})$ showed efficacy in modulating glucagon secretion from isolated rat islets (Figure 7), while no effect on insulin secretion was observed. Thus, the specificity of FGF-21 bioactivity remains to be further studied.

The administration of FGF-21 to diabetic $o b / o b$ and $d b / d b$ mice and obese $\mathrm{ZDF}$ rats led to significant lowering of circulating glucose and triglycerides, as well as a reduction in fasted insulin levels and improved glucose clearance during an OGTT (Figure 4, A-H). All these effects were observed after at least 3 days of injections and were more pronounced after 7 days of administration. Since no changes in levels of fed and fasted glucose, circulating lipid levels, insulin levels, and glucose disposal during OGTT were observed after a single s.c. injection of FGF-21, it appears that beneficial FGF-21-dependent effects require that animals be exposed to the protein multiple times. However, once the reduction in circulating glucose was achieved, FGF-21-induced changes were sustained for at least 24 hours (Figure 4F). Thus, despite its short elimination half-life, FGF-21 induced an extended pharmacodynamic effect in these diabetic animals. Taken together, these observations are remarkable in highlighting the difference in time action between FGF-21 and insulin.

Although potent in correcting elevated glucose levels in $o b / o b$ and $d b / d b$ mice, FGF-21 did not induce hypoglycemia in normal or diabetic rodents in either fasted or fed states (Figure 4, A, C-F, and H) at efficacious or significantly higher doses, and no hypoglycemia was seen in fasted FGF-21-transgenic mice (Table 1). This further distinguishes the effects of FGF-21 from those of insulin, which induced a significant reduction of blood glucose in lean animals (Figure 4E). Moreover, FGF-21 did not affect food intake or body weight/composition of diabetic or lean mice and rats over the course of 2 weeks of administration (doses ranging from $25 \mu \mathrm{g} / \mathrm{kg} / \mathrm{d}$ to $8 \mathrm{mg} / \mathrm{kg} / \mathrm{d}$ ).

\section{Figure 6}

FGF-21-transgenic mice are resistant to diet-induced weight gain and fat accumulation. (A) Average cumulative weight gain of FGF-21transgenic and wild-type mice fed an HFHC diet for 15 weeks. The values $( \pm$ SE) shown are the average of the measurements of at least 5 animals in a group. $P<0.05$ for all time points for male $(M)$ wild-type versus FGF-21-transgenic mice; and for weeks 6-15 for female (F) wild-type versus FGF-21-transgenic mice. (B) Body composition of male FGF-21-transgenic and wild-type mice before and after feeding on an HFHC diet for 15 weeks as determined by nuclear magnetic resonance. $P<0.002$ for lean and fat mass of FGF-21-transgenic versus wild-type mice.

Further insights into the FGF-21 mechanism of action can be gleaned from the phenotype of FGF-21-transgenic mice. These animals are viable and are not metabolically distinguishable from wild-type littermates at 2 months of age. However, they appeared to be resistant to the age-related impairment of glucose metabolism since they had lower plasma glucose levels at 9 months (Table 1). Moreover, when challenged on HFHC diet for 15 weeks, FGF-21transgenic mice were resistant to diet-induced weight gain and fat accumulation (Figure 6, A and B), even though they consumed more food when the amounts were normalized to body weights (Table 1). We also observed lower levels of circulating leptin (Table 1). The reduction in leptin is consistent with lower adiposity in the transgenic animals and may be a primary cause of the increased food intake in the transgenic mice (31). However, these changes in feeding behavior are unlikely to have been induced by a direct effect of FGF-21, since no impact on food intake in rodents administered the protein was observed. There was also a decrease in circulating glucagon levels (Table 1), which is consistent with the in vitro observations made with rat pancreatic islets (Figure 7).

There was no evidence of poor nutrient absorption in FGF-21transgenic animals. Thus, another potential reason for the observed resistance to diet-induced obesity may be an effect of FGF-21 on energy expenditure. However, if this is true, it was not reflected in any changes in rectal body temperatures (Table 1). We speculate that FGF-21 overexpression may increase brown adipose tissue (BAT) activity, as we observed retention of BAT in the transgenic mice compared with wild-type controls (Figure 5A). Whether or not BAT activation may contribute to the effects of FGF-21 remains to be evaluated in future studies.

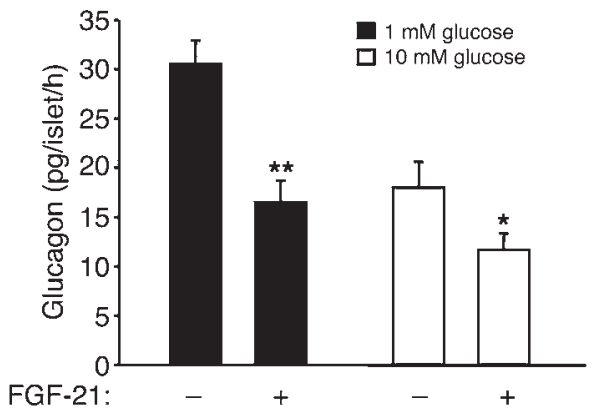

Figure 7

FGF-21 inhibits glucagon secretion in isolated rat pancreatic islets. The values ( \pm SE) shown are the average of 6 measurements. ${ }^{*} P<0.05$ and ${ }^{* *} P<0.02$ compared with vehicle controls. Islets were stimulated as indicated with FGF-21 in the presence of different glucose concentrations. 


\begin{tabular}{|c|c|c|c|}
\hline & Vehicle control & FGF-21 & $P$ value \\
\hline Glucagon (pM) & $252 \pm 40$ & $156 \pm 15$ & 0.04 \\
\hline Insulin (pM) & $2551 \pm 81.7$ & $2,344 \pm 46.3$ & 0.01 \\
\hline Adiponectin $(\mu \mathrm{g} / \mathrm{ml})$ & $12.3 \pm 0.82$ & $13.9 \pm 0.87$ & NS \\
\hline MCP-1 (pg/ml) & $111.8 \pm 18.9$ & $137.8 \pm 16.6$ & NS \\
\hline IL-6 (pg/ml) & $11.9 \pm 0.5$ & $12 \pm 0.7$ & NS \\
\hline TNF- $\alpha(p g / m l)$ & $11 \pm 0$ & $11.4 \pm 0.4$ & NS \\
\hline PAl-1 (pg/ml) & $2,104 \pm 339$ & $1,689 \pm 291$ & NS \\
\hline Amylin (pM) & $150 \pm 15$ & $113 \pm 10$ & NS \\
\hline Leptin (pM) & $12 \pm 3.3$ & $8.5 \pm 1.4$ & NS \\
\hline GLP-1 (pM) & $10.6 \pm 1.2$ & $13.3 \pm 0.9$ & NS \\
\hline
\end{tabular}

The values $( \pm \mathrm{SE})$ shown are the average of the measurements of 8 animals in a group. MCP-1, monocyte chemotactic protein-1; PAI-1, plasminogen activator inhibitor-1.

The FGF family member most closely related to FGF-21 is FGF-19, with $31 \%$ amino acid sequence identity between these 2 molecules (21). The phenotype of transgenic mice overexpressing FGF-19 $(12,13)$ is strikingly reminiscent of that of mice overexpressing FGF-21. However, we have uncovered a fundamental in vivo difference that clearly distinguishes these 2 molecules from each other. While transgenic mice overexpressing FGF-19 were resistant to high-fat dietinduced obesity, they also developed histologically detectable liver tumors. Furthermore, wild-type mice that were injected with FGF-19 for 6 days had an increase in hepatocellular proliferation (26). In contrast to FGF-19-overexpressing animals, FGF-21-transgenic mice did not form tumors in liver or show histological evidence of hyperplasia in any other tissue after 10 months of age (Figure 5C). Nevertheless, in order to further determine the potential of FGF-21 to stimulate liver mitogenicity in vivo, we administered FGF-21 to $d b / d b$ mice via ALZET pumps at an efficacious $11 \mu \mathrm{g} / \mathrm{kg} / \mathrm{h}$ dose (Figure 4C) for 8 weeks. Again, no evidence of hepatocellular proliferation or tumor formation was seen in FGF-21-infused mice as determined by $\mathrm{H} \& \mathrm{E}$ staining or proliferative cell nuclear antigen (PCNA) (32) immunohistochemistry (Figure 5D). Coupled with the lack of proliferation observed in vitro, the absence of tissue hyperplasia and activation of proliferative markers in FGF-21-treated and transgenic mice strongly suggests that FGF-21 is not mitogenic.

While our injection studies with FGF-21 in diabetic rodents clearly demonstrate that FGF-21 can lower glucose and lipids in circulation without inducing mitogenicity, hypoglycemia, or weight gain, the molecular mechanism by which FGF-21 functions is currently unclear. Despite the fact that FGF-21 and insulin induce similar functional outcomes in 3T3-L1 adipocytes (Figures 1-3) and FGF-21 has a positive impact on insulin sensitivity when administered to diabetic rodents or transgenic mice (Figure 4G and data not shown), it is unlikely that FGF-21 directly affects insulin-dependent pathways. Signaling and mitogenic profiles for these 2 proteins are different in vitro; no FGF-21-dependent tyrosine phosphorylation of insulin receptor was observed in 3T3-L1 adipocytes (data not shown); and there is no apparent interference between FGF-21 and insulin actions in glucose uptake assay (Figure 1C). Moreover, the pharmacology observed in FGF-21-treated animals (absence of hypoglycemia and weight gain; extended time-action) further distinguishes this molecule from insulin, which thus rules out a possibility that FGF-21 functions as an insulin mimetic and/or sensitizer.

The beneficial in vivo effects of FGF-21 may be mediated through changes in circulating levels of endocrine-relevant hormones, in particular, adipokines, since adipocytes appear to be a target for FGF-21 bioactivity. To explore this possibility, we dosed $o b / o b$ mice s.c. once daily with $125 \mu \mathrm{g} / \mathrm{kg} /$ day of FGF-21 for 7 days and measured the levels of several secreted polypeptides in circulation (Table 2). While we were able to achieve a clear glucose-lowering effect in the study (Figure 4A), only insulin and glucagon levels were changed in a statistically significant manner. The reduction of insulin levels is consistent with our observation in $\mathrm{o} b / \mathrm{ob}$ mice during OGTT (Figure 4G) and is suggestive of improvements in insulin sensitivity in FGF-21-treated mice.

GLUT1 and glucagon potentially mediate the mode of action of FGF-21. We were able to detect FGF-21-dependent upregulation of GLUT1 message specifically in white fat upon bolus injection into $o b / o b$ mice (Figure 1G), which thus confirms our in vitro observations on 3T3-L1 adipocytes. The increase in GLUT1 may mechanistically be linked to FGF-21-dependent glucose lowering in diabetic rodents. Alternatively, or in concert, the glucose lowering effect of FGF-21 is likely to result from reduced glucagon secretion from pancreatic $\alpha$ cells, since FGF-21 inhibits glucagon release in vitro (Figure 7) and is lowered in FGF-21-transgenic mice (Table 1). The observation of glucagon lowering in FGF-21-injected $o b / o b$ mice (Table 2) further strengthens the hypothesis that this hormone is an important mediator of FGF-21 in vivo effects. There is accumulating evidence supporting a pathophysiological role of glucagon in the development and progression of type 2 diabetes. Basal glucagon is inappropriately elevated and its suppression is impaired following food consumption, which leads to increased hepatic glucose production and aggravation of the hyperglycemia associated with the disease $(33,34)$. Interestingly, attenuation of signaling through the glucagon receptor leads to normalization of plasma glucose and triglyceride levels in diabetic animals (35).

Despite substantial progress in understanding the pathophysiology of diabetes mellitus and the development of new drugs to treat diabetic patients, this disease remains a major health problem (36). New treatments are required that will allow an efficacious regulation of glycemia and reduce the risk of the side effects associated with current therapies $(27,37,38)$. Here we demonstrate that FGF-21, as a single agent, can be used to provide efficient and durable glucose control and triglyceride lowering in diabetic animals, without apparent mitogenicity, hypoglycemia, or weight gain. FGF-21 thus holds promise as an effective therapeutic agent for the treatment of diabetes.

\section{Methods}

Expression and purification of FGF-21. A pET30a vector was used to express human FGF-21 in the Escherichia coli strain BL21(DE3) (Novagen; EMD Biosciences Inc.). FGF-21 product accumulated in the insoluble fraction. Inclusion bodies were prepared by standard centrifugation method. We solubilized inclusion bodies by bringing granule pellets to 10 times the original volume in $50 \mathrm{mM}$ Tris-HCL, pH 9.0, $7 \mathrm{M}$ urea and homogenizing the material. The protein mixture was adjusted to $\mathrm{pH} 11$, stirred for 1 hour, readjusted to $\mathrm{pH}$ 9.0, and loaded onto a Q Sepharose Fast Flow (Amersham Biosciences). Anion-exchange (AEX) chromatography was done in $50 \mathrm{mM}$ Tris-HCl, pH 9.0, $7 \mathrm{M}$ urea, $1 \mathrm{mM}$ DTT and with a 0-400 $\mathrm{mM} \mathrm{NaCl}$ gradient elution. The eluted AEX pool was treated with $10 \mathrm{mM}$ DTT for 2 hours at room temperature and diluted 10 -fold with $10 \mathrm{mM}$ cysteine/7 $\mathrm{M}$ urea. The protein was refolded by dialysis against $20 \mathrm{mM}$ glycine, $\mathrm{pH}$ 9.0, for 48 hours, 
at $4^{\circ} \mathrm{C}$. Further purification was carried out with reversed-phase highperformance liquid chromatography (RP-HPLC) performed with a Grace Vydac $\mathrm{C} 18$ column run in $\mathrm{H}_{2} \mathrm{O} / 0.1 \%$ trifluoroacetic acid/acetonitrile mobile phase with a $0-50 \%$ acetonitrile gradient; size-exclusion chromatography on Superdex 75 (Amersham Biosciences) in PBS, pH 7.4; and AEX chromatography on MonoQ (Amersham Biosciences) in $50 \mathrm{mM}$ Tris, $\mathrm{pH}$ 8.0, with 0-300 $\mathrm{mM} \mathrm{NaCl}$ gradient. The final FGF-21 pool was dialyzed into PBS, $\mathrm{pH} 7.4$, sterile filtered, and stored at $-80^{\circ} \mathrm{C}$.

Cell culture, adipocyte differentiation, glucose uptake, and mitogenicity experiments. 3T3-L1, NIH 3T3, BALB/c 3T3, 4MBr5, and clone 9 cells were from American Type Culture Collection; HMECs and HUVECs from Clonetics Corp.; and human primary preadipocytes from Zen-Bio Inc. A previously described 3T3-L1 adipocyte differentiation protocol (39) was adapted for Cytostar T 96-well plates (Amersham Biosciences). 3T3-L1 fibroblasts were seeded at 25,000 cells/well density; the differentiation was induced 2 days later in DMEM supplemented with $10 \% \mathrm{FBS}, 0.25 \mu \mathrm{M}$ dexamethasone, $0.5 \mathrm{mM}$ 3-isobutyl-1-methylxanthine, and $5 \mu \mathrm{g} / \mathrm{ml}$ insulin (48 hours); and the medium was changed to DMEM $/ 10 \% \mathrm{FBS} / 5 \mu \mathrm{g} / \mathrm{ml}$ insulin (48 hours). Thereafter, the cells were incubated for an additional 9-20 days in DMEM/10\% FBS (changed every other day). Primary human adipocytes were seeded in Cytostar T 96-well plates at 15,000 cells/well density; differentiated in Adipocyte Medium (AM; Zen-Bio Inc.) with 0.05 mM IBMX, $0.1 \mu \mathrm{M}$ dexamethasone, $10 \mathrm{nM}$ insulin, and $1 \mu \mathrm{M}$ rosiglitazone (14 days); and thereafter kept in AM (changed every other day). For glucose uptake, adipocytes were starved for 3 hours in DMEM/0.1\% BSA, stimulated with FGF-21 for 24 hours, and washed twice with KRP buffer (15 mM HEPES, pH 7.4, $118 \mathrm{mM} \mathrm{NaCl}$, $4.8 \mathrm{mM} \mathrm{KCl}, 1.2 \mathrm{mM} \mathrm{MgSO}_{4}, 1.3 \mathrm{mM} \mathrm{CaCl}_{2}, 1.2 \mathrm{mM} \mathrm{KH}_{2} \mathrm{PO}_{4}, 0.1 \% \mathrm{BSA}$ ), and $100 \mu \mathrm{l}$ of KRP buffer containing 2 -deoxy-D- $\left[{ }^{14} \mathrm{C}\right]$ glucose (2-DOG) $(0.1 \mu \mathrm{Ci}, 100 \mu \mathrm{M})$ was added to each well. Control wells contained $100 \mu \mathrm{l}$ of KRP buffer with 2-DOG $(0.1 \mu \mathrm{Ci}, 10 \mathrm{mM})$ to monitor for nonspecificity. The uptake reaction was carried out for 1 hour at $37^{\circ} \mathrm{C}$, terminated by addition of cytochalasin B $(20 \mu \mathrm{M})$, and measured using Wallac 1450 MicroBeta counter (Perkin Elmer). For mitogenicity experiments, cells were grown to confluence, starved for 24 hours in DMEM/0.5\% FCS, stimulated for 18 hours, and incubated with $0.25 \mu \mathrm{Ci}\left[{ }^{3} \mathrm{H}\right]$ thymidine per well for 2 hours. Cell lysates were then harvested and counted.

RNA extraction, cDNA synthesis, quantitative PCR. We used an RNeasy 96 Kit (QIAGEN Inc.) to extract RNA from cells and TRIzol reagent (Invitrogen Corp.) to extract RNA from tissues. We performed reverse transcription using a SuperScript First-Strand Synthesis kit (Invitrogen Corp.). The forward and reverse primer sequences for GLUT1 were 5'GCCCCCAGAAGGTTATTGA- 3 ' and $5^{\prime}$-CGTGGTGAGTGTGGTGGATG$3^{\prime}$, respectively. The probe sequence was $5^{\prime}$-TTCTACAATCAAACATGGAACCACCGCA-3'. To normalize for differences in the amount of total RNA added to each reaction, we performed amplification of $18 \mathrm{~S}$ ribosomal RNA as an endogenous control.

Immunoblotting, immunoprecipitation, and multiplex assays. 3T3-L1 adipocytes were starved for 18 hours, stimulated with FGF-21 ( $1 \mu \mathrm{g} / \mathrm{ml})$ for 10 minutes or for the indicated times (Figures $1 \mathrm{~F}$ and $2 \mathrm{~A}$ ), and lysed (40), and soluble fractions were analyzed. Antibodies were: anti-phospho-MAPK (Thr202/Tyr204), anti-MAPK, anti-phospho-FRS-2 (Y196) (Cell Signaling Technology); anti-FRS-2 (H-91) and anti-FGFR-2 (C-17) (Santa Cruz Biotechnology Inc.); anti-phosphotyrosine (4G10; Upstate); rabbit polyclonal: anti-GLUT1 against the 29 C-terminal amino acids of the human sequence, anti-GLUT4 (41), and anti-FGFR-1 against the 15 C-terminal amino acids of the mouse sequence. For immunodetection, goat anti-mouse and antirabbit HRP conjugates (Bio-Rad Laboratories) and ECL detection system (Amersham Biosciences) were used. We measured hormone and adipokine levels in circulation of vehicle-treated and FGF-21-injected $o b / o b$ mice using Multiplex assay kits from LINCO Research Inc.
Tissue preparation, bistology analysis, and immunostaining. Tissues were fixed overnight in zinc-buffered formalin and then transferred to $70 \%$ ethanol prior to processing through paraffin. Five-micrometer sections were stained with $\mathrm{H} \& \mathrm{E}$. Adjacent 5-micrometer sections were placed on positively charged slides. The slides were then baked overnight at $60^{\circ} \mathrm{C}$ in an oven and then deparaffinized in xylene and rehydrated through graded alcohols to water. Antigen retrieval was performed by immersing the slides in Target Retrieval Solution for 20 minutes at $90^{\circ} \mathrm{C}$, cooling at $25^{\circ} \mathrm{C}$ for 10 minutes, and washing in water; we then proceeded with immunostaining. All subsequent staining steps were performed on the Autoimmunostainer; incubations and all washes were done at $25^{\circ} \mathrm{C}$ in $50 \mathrm{mM}$ Tris- $\mathrm{HCl}, \mathrm{pH} 7.4$, containing $0.05 \%$ Tween-20. Slides were blocked with protein blocking solution for 25 minutes, and the PCNA antibody (PC10 clone) was incubated at a dilution of 1:10 for 1 hour. A biotinylated antibody plus streptavidin-HRP kit was then applied and followed with 3,3'-diaminobenzidine (DAB) staining. The slides were briefly counterstained with hematoxylin. All immunoreagents and the Autoimmunostainer were from Dako Corp.

Pancreatic islet isolation and hormone release studies. Pancreatic islets from male Wistar rats (200 g; Harlan Winkelmann GmbH) were isolated and cultured as described previously (35). For measurements of glucagon and insulin release, islets were starved in Earle's balanced salt solution medium containing $1 \mathrm{mM}$ glucose for 30 minutes. Groups of 10 (glucagon) or 3 (insulin) islets were selected and transferred into $0.3 \mathrm{ml}$ of EBSS medium with tested compounds. Islets were further incubated for 90 minutes at $37^{\circ} \mathrm{C}$ with vehicle or FGF-21 $(1 \mu \mathrm{g} / \mathrm{ml})$, supernatants were collected, and hormone content was measured.

In vivo protocols. The protocols used in these studies were approved by the Eli Lilly Research Laboratories Institutional Animal Care and Use Committee. Mice were maintained in a controlled environment $\left(21 \pm 2^{\circ} \mathrm{C}, 50-60 \%\right.$ humidity, 12-hour light-dark cycle, lights on at $6 \mathrm{am}$ ). Male $o b / o b$ and $d b / d b$ mice were from Harlan Teklad, fed Purina 5008 Chow, and had free access to food and water. FGF-21 was administered by s.c. injection in saline. For OGTT, the animals were fasted 16 hours and challenged by an oral glucose load $(2.5 \mathrm{~g} / \mathrm{kg}) 1$ hour after the last injection. Blood samples were taken from conscious, fed animals by tail snip, and glucose and plasma triglyceride levels were determined using Precision G Blood Glucose Testing System (Abbott Laboratories) and Hitachi 912 Clinical Chemistry analyzer (Roche Diagnostics Corp.), respectively. Insulin and leptin levels were determined with murine ELISA kits (Crystal Chem Inc.).

Male obese or lean ZDF rats were obtained from Charles River Laboratories Inc. and housed singly in a humidity and temperature-controlled environment $\left(21 \pm 2^{\circ} \mathrm{C}, 50\right.$-60\% humidity, 12 -hour light-dark cycle, lights on at $6 \mathrm{am}$ ) with free access to food (Purina 5008 Chow) and water for 2 weeks prior to start of the experiment. The day before the study, all animals were tail bled, and plasma glucose was analyzed on a Hitachi 912 Clinical Chemistry analyzer (Roche Diagnostics Corp.). Rats were then randomized based on their glucose levels and body weights and placed into groups. Animals were dosed with vehicle (0.9\% saline), insulin (Humulin; Eli Lilly and Company), or FGF-21 for 7 continuous days of twice-daily administration. On days 3 and 7, fed rats were bled (by tail snip) at 1-hour after administration of the last dose, and plasma glucose was assayed as described above.

The human apoE promoter including its hepatic control region (42) was used to express the human FGF-21 cDNA in transgenic mice. The transgenic vector was linearized and microinjected into C57BL/6NTac eggs by standard methods (43). We identified transgenic mice by PCR using transgene-specific primers and confirmed transgene expression by real-time quantitative PCR on RNA isolated from livers as well as by ELISA on plasma obtained from the transgenic mice using an FGF-21 polyclonal antibody.

For high-fat feeding, all mice were housed individually in Micro-Isolator cages (Lab Products Inc.) and maintained from age 24 days on high-fat TD95217 chow (40\% fat; Harlan Teklad) with free access to food and water. 
A wide-line nuclear magnetic resonance (NMR) instrument (Bruker BioSpin Corp.) was used to quantify tissue mass. Unanesthetized mice were placed into a 5 -cm diameter glass cylinder that was lowered into the instrument. Data was obtained for 4.5 minutes ( 3 determinations at 1.5 -minute intervals) and analyzed by the manufacturer's software. The mean of triplicate determinations for apparent muscle mass, fat mass, and free water mass was calculated for each mouse. Coefficient of variation of all 3 masses determined for a live moving mouse was less than $3 \%$.

\section{Acknowledgments}

We wish to thank P. Atkinson, G. Kelly, T. Black, B. Pies, and B. Strifler for supporting FGF-21 protein production; D. Bruce Baldwin for the generation of FGFR-FC-fusion constructs; S. Bright and J. Dunbar for FGF-21/FGFR binding experiments; S. Sissons and W. Roell for assistance with glucose uptake and proliferation assays; N. Fox and K. Brune for generation of FGF-21transgenic animals; K. Coble for help with pharmacokinetic studies; M. Brenner and A. Efanov for assistance with glucagon secretion experiments; D. Ballard and K. Mintze for supporting histochemistry work; C. Shrake for in vivo assistance; J. Manetta and L. Slieker for generation of polyclonal anti-FGF-21, antiGLUT1, and anti-GLUT4 antibodies; A. Klip (Hospital for Sick Children, Toronto, Ontario, Canada) for L6-GLUT-4myc cells; J. Caro, S. Jacobs, and G. Etgen for critically reading the manuscript; and T. Bumol, B. Grinnell, A. Glasebrook, R. Smith, and S. Taylor for helpful discussions.

Received for publication October 12, 2004, and accepted in revised form March 23, 2005.

Address correspondence to: Alexei Kharitonenkov, Lilly Research Laboratories, Division of Eli Lilly and Company, Indianapolis, Indiana 46285, USA. Phone: (317) 276-0091; Fax: (317) 277-2934; E-mail: a.kharch@lilly.com.
1. McKeehan, W.L., Wang, F., and Kan, M. 1998. The heparan sulfate-fibroblast growth factor family: diversity of structure and function. Prog. Nucleic Acid Res. Mol. Biol. 59:135-176.

2. Galzie, Z., Kinsella, A.R., and Smith, J.A. 1997. Fibroblast growth factors and their receptors. Biochem. Cell Biol. 75:669-685.

3. Powers, C.J., McLeskey, S.W., and Wellstein, A. 2000. Fibroblast growth factors, their receptors and signaling. Endocr. Relat. Cancer. 7:165-197.

4. Hogan, B.L. 1999. Morphogenesis. Cell. 96:225-233.

5. Ornitz, D.M., and Itoh, N. 2001. Fibroblast growth factors. Genome Biol. 2:reviews3005. doi:10.1186/ gb-2001-2-3-reviews3005.

6. Sakaue, H., et al. 2002. Requirement of fibroblast growth factor 10 in development of white adipose tissue. Genes Dev. 16:908-912.

7. Bhushan, A., et al. 2001. FGF10 is essential for maintaining the proliferative capacity of epithelial progenitor cells during early pancreatic organogenesis. Development. 128:5109-5117.

8. Ohuchi, H., et al. 2000. FGF10 acts as a major ligand for FGF receptor 2 IIIb in mouse multiorgan development. Biochem. Biophys. Res. Commun. 277:643-649.

9. Konishi, M., Micami, T., Yamasaki, M., Miyake, A., and Itoh, N. 1999. Fibroblast growth factor-16 is a growth factor for embryonic brown adipocytes. J. Biol. Chem. 255:12119-12122.

10. Nishimura, T., Utsonomiya, Y., Hoshikawa, M., Ohuchi, H., and Itoh, N. 1999. Structure and expression of a novel human FGF, FGF-19, expressed in the fetal brain. Biochim. Biophys. Acta. 1444:148-151.

11. Xie, M.H., et al. 1999. FGF-19, a novel fibroblast growth factor with unique specificity for FGFR4. Cytokine. 11:729-735.

12. Tomlinson, E., et al. 2002. Transgenic mice expressing human fibroblast growth factor-19 display increased metabolic rate and decreased adiposity. Endocrinology. 143:1741-1747.

13. Fu, L., et al. 2004. Fibroblast growth factor 19 increases metabolic rate and reverses dietary and leptin deficient diabetes. Endocrinology. 145:2594-2603.

14. Kim, I., Moon, S., Yu, K., Kim, U., and Koh, G.Y. 2001. A novel fibroblast growth factor receptor-5 preferentially expressed in the pancreas. Biochim. Biophys. Acta. 1518:152-156.

15. Sleeman, M., et al. 2001. Identification of a novel fibroblast growth factor receptor, FGFR5. Gene. 271:171-182.

16. Hart, A.W., Baeza, N., Apelqvist, Å., and Edlund,
H. 2000. Attenuation of FGF-signalling in mouse $\beta$-cells leads to diabetes. Nature. 408:864-868.

17. Revest, J.M., et al. 2001. Fibroblast growth factor receptor 2 IIIb acts upstream of Shh and FGF4 and is required for limb bud maintenance but not for the induction of FGF8, FGF10, Msx1, or BMP4. Dev. Biol. 231:47-62.

18. Celli, G., LaRochelle, W.J., Mackem, S., Sharp, R., and Merlino, G. 1998. Soluble dominant-negative receptor uncovers essential roles for fibroblast growth factors in multi-organ induction and patterning. EMBO J. 17:1642-1655.

19. Elghazi, L., Cras-Meneur, C., Czernichow, P., and Scharfmann, R. 2002. Role for FGFR2IIIb-mediated signals in controlling pancreatic endocrine progenitor cell proliferation. Proc. Natl. Acad. Sci. U.S. A. 99:3884-3889.

20. Yu, C., et al. 2000. Elevated cholesterol metabolism and bile acid synthesis in mice lacking membrane tyrosine kinase receptor FGFR4. J. Biol. Chem. 275:15482-15489.

21. Nishimura, T., Nakatake, Y., Konishi, M., and Itoh, N. 2000. Identification of a novel FGF, FGF-21, preferentially expressed in the liver. Biochim. Biophys. Acta. 1492:203-206.

22. Ueyama, A., Yaworsky, K.L., Wang, Q., Ebina, Y., and Klip, A. 1999. GLUT-4myc ectopic expression in L6 myoblasts generates a GLUT-4-specific pool conferring insulin sensitivity. Am. J. Physiol. 277:572-578.

23. Lin W.-W., and Hsu, Y.-W. 2000. Cycloheximideinduced $\mathrm{CPLA}_{2}$ activation is via the MPK-1 down-regulation and ERK activation. Cell. Signal. 12:457-461.

24. Kouhara, H., et al. 1997. A lipid-anchored GRB2binding protein that links FGF-receptor activation to the Ras/MAPK signalling pathway. Cell. 89:693-702.

25. Schlessinger, J. 2000. Cell signalling by receptor tyrosine kinases. Cell. 103:211-225.

26. Nicholes, K., et al. 2002. A mouse model of hepatocellular carcinoma. Ectopic expression of fibroblast growth factor 19 in skeletal muscle of transgenic mice. Am. J. Pathol. 160:2295-2307.

27. Moller, D.E. 2001. New drug targets for type 2 diabetes and the metabolic syndrome. Nature. 414:821-827.

28. Chen, C., et al. 2003. An integrated functional genomics screening program reveals a role for BMP-9 in glucose homeostasis. Nat. Biotechnol. 21:294-301.

29. Shepherd, P.R., and Khan, B.B. 1999. Glucose transporters and insulin action. N. Eng. J. Med. 341:248-257.
30. Coulier, F., et al. 1997. Of worms and men: an evolutionary perspective on the fibroblast growth factor (FGF) and FGF receptor families. J. Mol. Evol. 44:43-56.

31. Frederich, R.C., et al. 1995. Leptin levels reflect body lipid content in mice: evidence for diet-induced resistance to leptin action. Nat. Med. 1:1311-1314.

32. Linden, M.D., Torres, F.X., Kubus, M.S., and Zarbo, R.J. 1992. Clinical application of morphologic and immunocytochemical assessments of cell proliferation. Am. J. Clin. Pathol. 97:S4-S13.

33. Reaven, G.M., Chen, Y.D., Golay, A., Swislocki, A.L., and Jaspan, J.B. 1987. Documentation of hyperglucagononemia throughout the day in nonobese and obese patients with noninsulin-dependent diabetes mellitus. J. Clin. Endocrinol. Metab. 64:106-110.

34. Shah, P., et al. A. 2000. Lack of suppression of glucagon contributes to postprandial hyperglycemia in subjects with type 2 diabetes mellitus. J. Clin. Endocrinol. Metab. 85:4053-4059.

35. Sloop, K.W., et al. 2004. Hepatic and glucagon-like peptide-1-mediated reversal of diabetes by glucagon receptor antisense oligonucleotide inhibitors. J. Clin. Invest. 113:1571-1581. doi:10.1172/ JCI200420911.

36. Zimmet, P., Alberti, K.G., and Shaw, J. 2001. Global and societal implications of the diabetes epidemic. Nature. 414:782-787.

37. Taylor, S.I. 1999. Deconstructing type 2 diabetes. Cell. 97:9-12.

38. Saltiel, A.R. 2001. New perspectives into the molecular pathogenesis and treatment of type 2 diabetes. Cell. 104:517-529.

39. Cheatham, B., et al. 1994. Phosphatidylinositol 3 -kinase activation is required for insulin stimulation of Pp70 S6 kinase, DNA synthesis, and glucose transporter translocation. Mol. Cell. Biol. 14:4902-4911.

40. Kharitonenkov, A., et al. 1997. A family of proteins that inhibit signalling through tyrosine kinase receptors. Nature. 386:181-186.

41. Slieker, L.J., et al. 1992. Glucose transporter levels in tissues of spontaneously diabetic Zucker fa/fa rat (ZDF/drt) and viable yellow mouse (Avy/a). Diabetes. 41:187-193.

42. Allan, C.M., Walker, D., and Taylor, J.M. 1995. Evolutionary duplication of a hepatic control region in the human apolipoprotein E gene locus. J. Biol. Chem. 270:26278-26281.

43. Hogan, B., Beddington, R., Constantini, F., and Laci, E. 1994. Manipulating the mouse embryo. Cold Spring Harbor Press. Cold Spring Harbor, New York, USA. 487 pp. 